\title{
Insight into Macular Vascular Changes Seen in Retinitis Pigmentosa Using OCTA
}

\section{Lindsay $\mathrm{BH}^{*}$ and Julie R}

Nova Southeastern University College of Optometry, USA

*Corresponding author: Lindsay Baker Howse, Nova Southeastern University College of Optometry 3200 S, University Drive Fort Lauderdale, FL 33328, USA, Tel: 801-9134687; E-mail: drlindsayhowse@gmail.com

\section{Case Report}

Volume 2 Issue 2

Received Date: July 15, 2017

Published Date: July 24, 2017

\section{Abstract}

Statement of Significance: Optical Coherence Tomography Angiography (OCTA) allows for compete assessment of macular vascular density of various stages of retinitis pigmentosa.

Purpose: Retinitis pigmentosa (RP) is an inherited retinal dystrophy characterized by progressive degeneration of the rod photoreceptor cells with eventual degeneration of cone photoreceptors as well. The advent of Optical Coherence Tomography Angiography (OCTA) has provided new insight into the pathophysiology behind the disease including disorganization within the inner retina that coincides with morphological changes within the outer retina and photoreceptors. Furthermore, retinal vascular alterations within the inner retina appear to be directly correlated with visual function in affected patients.

Case Report: Three patients with varying stages of RP are presented. Patients were evaluated and categorized based on visual acuity, funduscopic findings, electrodiagnostics, OCT and OCTA. Vascular density within the retinal and choroidal vessels was evaluated with the Spectral-Domain Optical Coherence Tomography Angiography with XR Avanti (Optovue, Inc., Fremont, CA, USA). Degree of vascular reduction within the inner retinal SCP (superficial capillary density) and DCP (deep capillary density) was directly correlated with stage of disease and visual function.

Conclusions: Vascular alterations including arterial narrowing, vessel attenuation and changes in blood flow have been well documented in RP patients. OCTA now provides a 3-dimensional volumetric analysis of retinal blood flow throughout all layers of the retina allowing for direct correlation between structure and vascular integrity. The correlation between inner retinal integrity, vascular structure and visual function can be optimally examined with the integration of OCTA and treatment modalities can be appropriately aimed at targeting viable retinal photoreceptors.

Keywords: Retinitis Pigmentosa; Optical Coherence Tomography Angiography; Funduscopic; Choroidal; Nyctalopia 


\section{Open Access Journal of Ophthalmology}

Abbreviations: OCTA: Optical Coherence Tomography Angiography; RP: Retinitis Pigmentosa; SCP: Superficial Capillary Density; DCP: Deep Capillary Density

\section{Introduction}

Retinitis Pigmentosa (RP) is defined as a group of rare, hereditary retinal diseases that results in progressive loss of photoreceptors. Patients typically present with nyctalopia and progressive loss of peripheral vision [1]. For many years, the focus of Retinitis Pigmentosa (RP) has been on understanding the pathophysiology of the disease and identifying the various structural abnormalities that manifest throughout the disease progression including arteriole attenuation, waxy optic nerve pallor, bone spicule formation, posterior subcapsular cataracts and macular mottling. OCT angiography (OCTA) is a new, innovative, non-invasive imaging modality that allows for advanced imaging and analysis of the macular vascular changes associated with RP that have not been previously reported. These findings have provided additional insight into the pathophysiology behind the disease suggesting involvement at the level of the inner retina. We present three cases of RP at varying stages to highlight the changesin macular vascular density throughout the retinal and choroidal layers as the disease progresses.

\section{Case Report}

\section{Case 1}

An 18-year-oldAfricanAmerican male presented with a history of unknown retinal dystrophy, presumed RP. Two years ago, a prior doctor had suspected RP based on mild clinical signs, but was unable to confirm without ERG. The patient had no complaints of decreased night vision or peripheral vision. After probing, the patient reported slight delay in visual recovery after looking at bright lights. The patient denied a family history of RP. His systemic history was unremarkable and he was not taking any medications. Best corrected visual acuities were 20/20 OD, OS. Pupils, confrontations, extraocular motility and color vision were found to be normal. Intraocular pressure measured $11 \mathrm{mmHg}$ OD, OS. Anterior segment findings were unremarkable, with no posterior subcapsular cataracts. Both eyes exhibited similar findings upon fundus evaluation. The optic nerves were pink and healthy, C/D ratio of 0.3 , with no waxy pallor. The macula was intact, with no hemorrhaging, edema or mottling. Retinal vasculature demonstrated slight vessel attenuation. In addition, there was mild bone spicule formation in the periphery and mid-periphery.
SD-OCT of the macula appeared healthy with no apparent structural abnormalities. Vascularization of the retinal layers was visualized and segmented using OCT Angiography. In addition, objective quantification of vessel density was evaluated for each eye using the AngioVue software. Analysis of the angiograms revealed decreased retinal vascular perfusion and capillary density in both the superficial capillary plexus (SCP) and deep capillary plexus (DCP) with no apparent abnormality at the choriocapillaris. Careful evaluation of the vessel density maps revealed more significant loss at the level of the SCP than the DCP (Figure 1). Despite the relatively quiescent nature of the fundus appearance and SD-OCT, OCTA was able to detect and localize irregularities in vascular flow within the inner retina in this patient with mild disease.

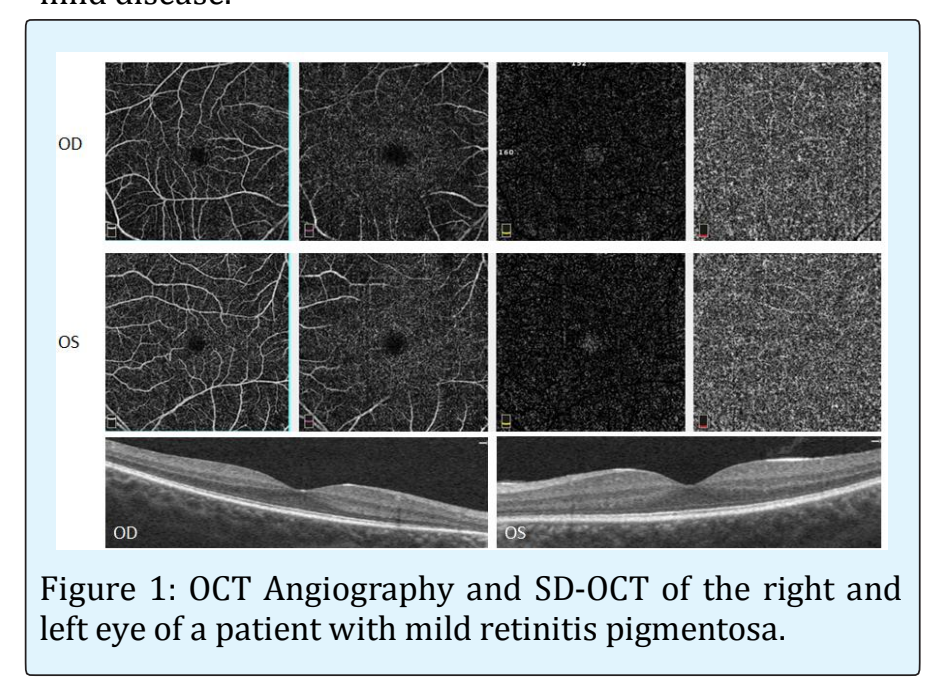

\section{Case 2}

A 27-year-old Hispanic male presented with chief complaint of diminution of vision in both eyes and a history of RP. His systemic history was unremarkable and he was taking no medications. Best corrected visual acuities were 20/25- OD, OS. Pupils, confrontations, extraocular motility and color vision were found to be normal. Intraocular pressure was measured to be $14 \mathrm{mmHg}$ OD and $15 \mathrm{mmHg}$ OS. Anterior segment findings were unremarkable, with no posterior subcapsular cataracts. Posterior segment findings of both eyes revealed mild pallor of the optic nerves with 0.3 round C/D ratio. The macula was intact, with no hemorrhaging, edema or mottling. There was a mild epiretinal membrane present in the macula right eye greater than left eye. The retinal vessels exhibited mild vessel attenuation. The periphery was flat and intact with bone spicule RPE clumping in all quadrants.

Significant findings on the SD-OCT include depiretinal 


\section{Open Access Journal of Ophthalmology}

membrane and slight disruption of the PIL (photoreceptor integrity line) in both eyes. OCT Are vealedan enlarged foveal avascular zone with abnormally low vascular density in the superficial and deep capillary plexus with more significant involvement of the deep capillary plexus with no apparent abnormality seen at the level of the choroid (Figure 2). With moderate RP, as seen with this patient, the DCP appears to be more significantly involved than the SCP, although both complexes are severely compromised [2].

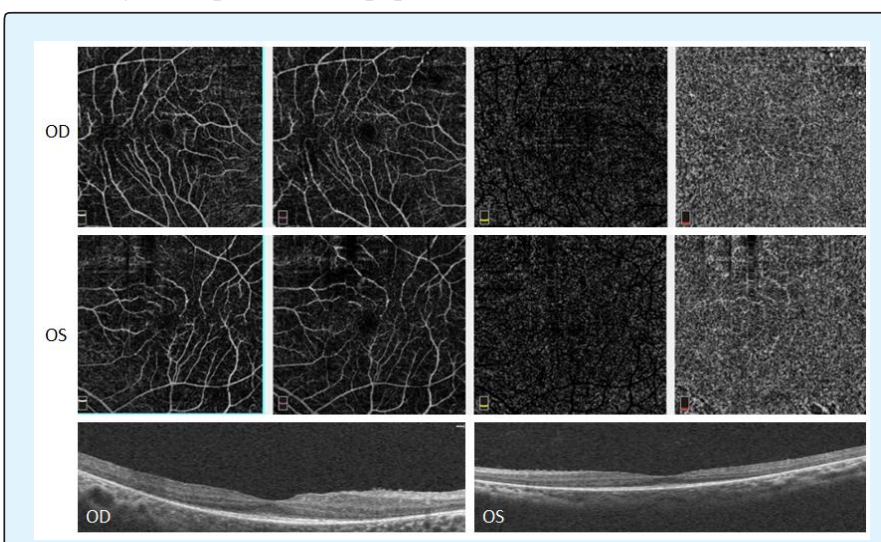

Figure 2: OCT Angiography and SD-OCT of the right and left eye of a patient with moderate retinitis pigmentosa.

\section{Case 3}

A 56-year-old Hispanic male presented with a chief complaint of blurry vision and decreased peripheral vision in both eyes. The patient was diagnosed with RP 20 years prior in Cuba. His systemic history was unremarkable and he was taking nomedications. The patient reported a family history of RP and Diabetes Mellitus type 2. Best corrected visual acuities were 20/60 OD and 20/50 OS. Extraocular motility was full, however confrontation fields were significantly reduced in all quadrants in both eyes. Pupils were normal with no APD. The patient failed the HRR color vision screening in both eyes. Intraocular pressure was measured to be $14 \mathrm{mmHg}$ OD, OS. Anterior segment findings revealed mild MGD, pinguecula, trace amount of pigment on the corneal endothelium, and PC IOL with 1+ diffuse PCO in both eyes. Significant posterior segment findings of both eyes included: waxy pallor of both optic nerves with $0.2 \mathrm{C} / \mathrm{D}$ ratio, retinal vascular attenuation, pigmentary changes and RPE mottling of the macula, and bone spicule RPE clumping in the periphery in all quadrants.

SD-OCT showed mild macular edema with cystic pockets of fluid in both eyes, but visual acuity was only mildly affected due to preservation of the PIL and ellipsoid zone at the fovea. OCTA showed decreased vascular density in the SCP and DCP with the left eye affected more than the right eye. Mild changes were observed at the level of the choriocapillaris, but most of the change was observed in the SCP and DCP with the DCP more severely impacted than the SCP (Figure 3).The OCTA density maps confirmed that severity of disease correlated with reduction in vascular density within the inner retinal SCP and DCP, with reduction in DCP directly related to visual function.

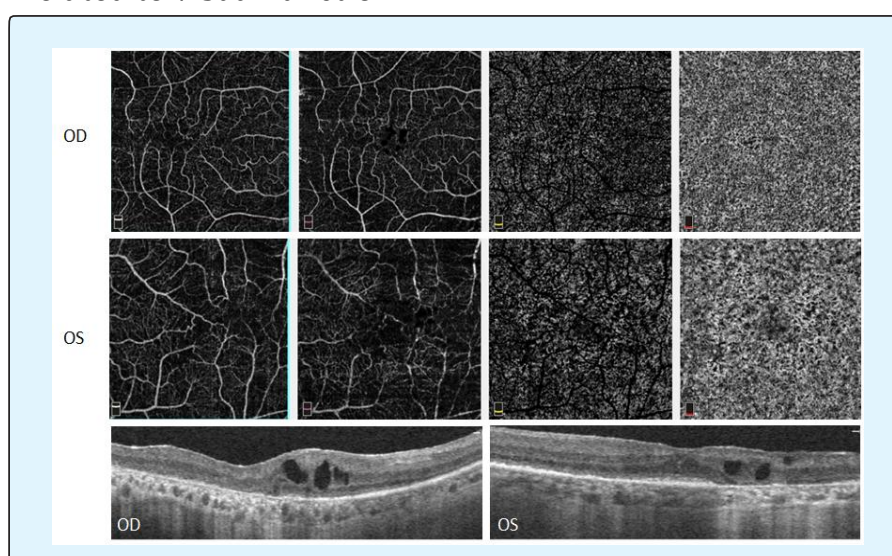

Figure 3: OCT Angiography and SD-OCT of the right and left eye of a patient with advanced retinitis pigmentosa.

\section{Discussion}

Retinitis pigmentosa (RP) is the term used to designate a group of inherited retinal disorders characterized by progressive loss of photoreceptors and retinal dysfunction resulting in deterioration of central vision. ${ }^{1}$ $\mathrm{RP}$ is characterized by classic symptomatology and clinical findings including nyctalopia, visual field constriction, bone spicule pigmentation and abnormal full-field electro-retinograms. RP affects the photoreceptor-RPE cell metabolic complex and begins with a loss of the rod photoreceptors as evidenced by the initial involvement of the equatorial and peripheral retina. Over time, the cone photoreceptors become involved resulting in progressive visual loss. Histopathological studies have identified shortening of the photoreceptor outer segments as the earliest identifiable finding in RP.(Liu) Typical clinical features of $\mathrm{RP}$ include peripheral retinal bone-spicule deposition, optic nerve pallor and retinal vessel attenuation. Loss of normal retinal vasculature is believed to be due to photoreceptor degeneration [3].

Abnormalities within the retinal vasculature as well as changes in ocular blood flow have been implicated in the 


\section{Open Access Journal of Ophthalmology}

pathogenesis of RP. In fact, fluorescein angiography (FA) and indocyanine green angiography (ICG) have illustrated the abnormal retinal and choroidal vasculature in RP noting increased dye transit time, narrowed vessel lumen, and reduced dye concentration [4]. In addition, electoretinogram (ERG) shows increased arteriovenous transit time and reduced blood flow velocity [5]. Since the advent of Optical Coherence Tomography (OCT), OCT has become the standard for assessing anatomical abnormalities via high resolution tomographic images. OCT is able to accurately assess the photoreceptor segments and thicknesses of retinal layers in RP patients and has shown a decrease in foveal thickness, interruption of the IS/OS junction, and reduction in choroidal thickness [5]. Correlations between the retinal structure and visual acuity have been identified in affected patients.

Historically, RP has been characterized as a disease of the photoreceptors with loss of the outer retinal cells. However, new research is identifying disorganization and damage to the inner retina including death of the retinal ganglion cells. Outer retinal involvement can occur as a result of chronic apoptosis of the ganglion cells and disorganization of the inner retina [6-8]. Optical coherence tomography angiography (OCTA) is a recently developed, new, noninvasive, contrast-free technique for imaging of the retinal and choroidal microvasculature. The technique uses the movement of red blood cells in the retinal vasculature to create an image of retinal blood flow. This allows for segmentation and evaluation of all retinal layers and capillary networks. Analysis of OCTA images in RP patients has confirmed the aforementioned involvement of the inner retinal layers in the pathogenesis of RP. It has been documented that photoreceptor damage leads to changes in the inner retinal tissue including the retinal ganglion cells. Despite varying hypotheses regarding the morphological changes to the inner retina, a direct correlation has been found between ganglion cell thickness and macular function in RP patients. Furthermore, it appears that the mfERG is negatively affected by apoptosis of the ganglion cells [9].

The resolution provided by OCTA has provided outstanding visualization of the superficial (SCP) and deep capillary (DCP) vascular networks within the inner retina and has been able to identify varying features that cannot be distinguished by classical FA. The superficial vascular plexus is located in the ganglion cell layer and in the nerve fiber layer. The deep vascular plexiare located in the inner nuclear layers and external plexiform layer. OCT angiography illustrates different morphological features of the retinal blood supply for the two plexi [10]. The vascular supply of the superficial plexus is characterized by multiple linear structures having a centripetal distribution converging towards the fovea. Secondary vessels arise from the main vessels forming a web-like pattern. The caliber of the vessels is uniform throughout the scan. The deep plexus is made up of in interwoven pattern of vessels that surround the foveal avascular zone in numerous horizontal and radial interconnections. The caliber of the vessels is also uniform throughout the scan $[11,12]$.

OCTA in patients with RP demonstrate alterations in the normal vasculature within the superficial and deep retinal layers as well as the throughout the choroid. The SCP and DCP vessel densities are reduced in patients with RP. It has been postulated that this reduction in blood flow occurs early on in the disease process and leads to eventual ischemia, retinal vessel damage and cell death involving both the inner and outer retinal space [9]. Furthermore, vessel density abnormalities at the level of the DCP appear to be directly related to macular function and visual potential. Analysis of the three patients presented in this manuscript suggest that severity of disease correlates with degree of reduction in vascular flow as seen on the segmented angiograms as well as with diminution in the vessel density map (Figure 4).

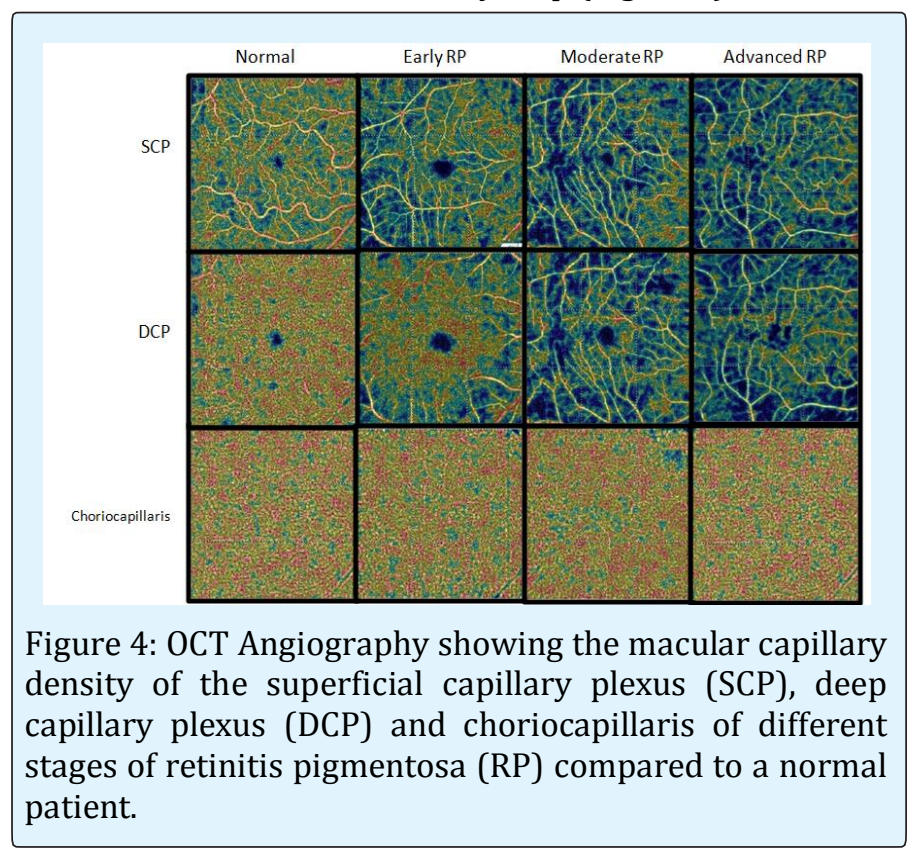

Since OCTA is able to identify and quantify loss of normal retinal vasculature in RP patients, it should be used to monitor disease progression in affected patients. Vascular changes may precede functional changes as seen 


\section{Open Access Journal of Ophthalmology}

on visual field testing and may thus play a pivotal role in staging and categorizing the disease.

\section{Conclusion}

In conclusion, OCTA has provided excellent insight into the microvascular changes occurring throughout the retina and choroid and has allowed for additional insight into the pathophysiology behind the disease. It appears that alterations in the vascular density and pattern of flow seen on angiogram correlates with disease severity. OCTA may exhibit signs of disease earlier and should be utilized to provide timely management of affected patients.

\section{References}

1. Rezaei KA, Zhang Q, Chen C, Chao J (2006) Retinitis Pigmentosa. Lancet 368: 1795-1809.

2. Liu G, Liu X, Li H, Du Q, Wang F (2016) Optical Coherence Tomographic Analysis of Retina in Retinitis Pigmentosa Patients. Ophthalmic Res 56(3): 111-122.

3. Zhang Q, Lee CS, Chao J, Chen CL, Thomas Z, et al. (2016) Wide-field Optical Coherence Tomography Based Microangiography for Retinal Imaging. Nature 6: 22017.

4. Merin S, Auerbach E (1976) Retinitis Pigmentosa. Surv Ophthalmol 122: 502-508.

5. Dhoot DS, Huo S, Yuan A, David X, Sunil S, et al. (2013) Evaluation of Choroidal Thickness in Retinitis Pigmentosa using Enhanced Depth Imaging Optical
Coherence Tomography. Br J Ophthalmol 97(1): 6669.

6. Stone JL, Barlow WE, Humayun MS, de Juan E, Milam AH (1992) Morphometric Analysis of Macular Photoreceptors and Ganglion Cells in Retinas with Retinitis Pigmentosa. Arch Ophthalmol 110(11): 1634-1639.

7. Jones BW, Pfeiffer RL, Ferrell WD, Watt CB, Marmor $M$, et al. (2016) Retinal Remodeling in Human Retinitis Pigmentosa. Exp Eye Res 150:149-165.

8. Saha S, Greferath U, Vessey KA, Grayden DB, Burkitt AN, et al. (2016) Changes in Ganglion Cells during Retinal Degeneration. Neuroscience 329: 1-11.

9. Toto L, Borrelli E, Mastropasqua R, Senatore A, Di Antonio L, et al. (2016) Macular Features in Retinitis Pigmentosa: Correlations Among Ganglion Cell Complex Thickness, Capillary Density, and Macular Function. Invest Ophthalmol Vis Sci 57(14): 63606366.

10. Spaide RF, Klancnik JM, Cooney MJ (2015) Retinal Vascular Layers Imaged by Fluorescein Angiography and Optical Coherence Tomography Angiography. JAMA Ophthalmol 133(1): 45-50.

11. Lumbroso B, Huang D, Jia Y (2015) Clinical Guide to Angio-OCT Non Invasive, Dye-less Oct Angiography. 1st (Edn.), New Delhi, Jaypee Brothers Medical Publisher, India.

12. Savastano MC, Lumbroso B, Rispoli M (2015) In Vivo Characterization of Retinal Vascularization Morphology using Optical Coherence Tomography Angiography. Retina 35(11): 2196-2203. 